Article

\title{
An Overview of the Hamilton-Jacobi Theory: the Classical and Geometrical Approaches and Some Extensions and Applications
}

\author{
Narciso Román-Roy
}

Citation: Román-Roy, N. An Overview of the Hamilton-Jacobi Theory: the Classical and Geometrical Approaches and Some Extensions and Applications. Mathematics 2021, 9, 85. https://doi.org/10.3390/ math9010085

Received: 29 November 2020 Accepted: 30 December 2020 Published: 3 January 2021

Publisher's Note: MDPI stays neutral with regard to jurisdictional claims in published maps and institutional affiliations.

Copyright: () 2021 by the authors. Licensee MDPI, Basel, Switzerland. This article is an open access article distributed under the terms and conditions of the Creative Commons Attribution (CC BY) license (https: / / creativecommons.org / licenses/by/4.0/).
Department of Mathematics, Universitat Politècnica de Catalunya, Ed. C3, Campus Nord, 08034 Barcelona, Spain; narciso.roman@upc.edu

\begin{abstract}
This work is devoted to review the modern geometric description of the Lagrangian and Hamiltonian formalisms of the Hamilton-Jacobi theory. The relation with the "classical" Hamiltonian approach using canonical transformations is also analyzed. Furthermore, a more general framework for the theory is also briefly explained. It is also shown how, from this generic framework, the Lagrangian and Hamiltonian cases of the theory for dynamical systems are recovered, as well as how the model can be extended to other types of physical systems, such as higher-order dynamical systems and (first-order) classical field theories in their multisymplectic formulation.
\end{abstract}

Keywords: Hamilton-Jacobi equations; Lagrangian and Hamiltonian formalisms; higher-order systems; classical field theories; symplectic and multisymplectic manifolds; fiber bundles

MSC: Primary: 35F21; 70H03; 70H05; 70H20; Secondary: 53C15; 53C80; 70G45; 70H50; 70 S05

\section{Introduction}

The Hamilton-Jacobi theory is a topic of interest in mathematical physics since it is a way to integrate systems of first-order ordinary differential equations (Hamilton equations in the standard case). The classical method in Hamiltonian mechanics consists of obtaining a suitable canonical transformation, which leads the system to equilibrium [1-4], and is given by its generating function. This function is the solution to the so-called Hamilton-Jacobi equation, which is a partial differential equation. The "classical" Hamilton-Jacobi problem consists of finding this canonical transformation. Because of its interest, the method was generalized in other kinds of physical systems, such as, for instance, singular Lagrangian systems [5] or higher-order dynamics [6], and different types of solutions have been proposed and studied $[7,8]$.

Nevertheless, in recent times, a lot of research has been done to understand the Hamilton-Jacobi equation from a more general geometric approach, and some geometric descriptions to the theory were done in Reference [9-13]. From a geometric way, the above mentioned canonical transformation is associated with a foliation in the the phase space of the system which is represented by the cotangent bundle $T^{*} Q$ of a manifold (the configuration manifold $Q$ ). This foliation has some characteristic geometric properties: it is invariant by the dynamics, transversal to the fibers of the cotangent bundle, and Lagrangian with respect to the canonical symplectic structure of $\mathrm{T}^{*} Q$ (although this last property could be ignored in some particular situations). The restriction of the dynamical vector field in $\mathrm{T}^{*} Q$ to each leaf $S_{\lambda}$ of this foliation projects onto another vector field $X_{\lambda}$ on $Q$, and the integral curves of these vector fields are one-to-one related. Hence, the complete set of dynamical trajectories are recovered from the integral curves of the complete family $\left\{X_{\lambda}\right\}$ of all these vector fields in the base. These geometric considerations can be done in an analogous way in the Lagrangian formalism; hence, this geometrical picture of the 
Hamilton-Jacobi theory can be also stated for this formalism. The geometric HamiltonJacobi problem consists of finding this foliation and these vector fields $\left\{X_{\lambda}\right\}$.

Following these ideas, the Lagrangian and Hamiltonian versions of the HamiltonJacobi theory, for autonomous and non-autonomous mechanical systems, was formulated in another geometrical way in Reference [14]. The foundations of this geometric generalization are similar to those given in Reference $[9,15]$. Later, this framework was used to develop the Hamilton-Jacobi theory for many other kinds of systems in physics. For instance, other applications of the theory are to the case of singular Lagrangian and Hamiltonian systems [16-19], higher-order dynamical systems [20,21], holonomic and non-holonomic mechanics $[19,22-27]$, and control theory [28,29]. The theory is also been extended for dynamical systems described using other geometric structures, such as Poisson manifolds [18,30], Lie algebroids [31,32], contact manifolds (which model dissipative systems) [33,34], and other geometric applications and generalizations: [35-37]. Furthermore, in Reference [38,39], the geometric discretization of the Hamilton-Jacobi equation was analyzed. Finally, the Hamilton-Jacobi theory is developed for the usual covariant formulations of first-order classical field theories, the $k$-symplectic and $k$-cosymplectic $[40,41]$ and the multisymplectic ones [42,43], for higher-order field theories [44,45], for the formulation in the Cauchy data space [46], and for partial differential equations in general [47,48].

This review paper is devoted, first of all, to present, in Section 2, the foundations of this modern geometric formulation of the Hamilton-Jacobi theory, starting from the most general problem and explaining how to derive the standard Hamilton-Jacobi equation for the Hamiltonian and the Lagrangian formalisms of autonomous mechanics. After this, the notion of complete solution allows us to establish the relation with the "classical" Hamilton-Jacobi theory based on canonical transformations, which is summarized in Section 3, where this relation is also analyzed (this topic had been already discussed in Reference [48]). We also present briefly a more general geometric framework for the Hamilton-Jacobi theory which was stated in Reference [36], from which we can derive the majority of the applications of the theory to other kinds of physical systems, including the case of autonomous dynamical systems. This is done in Section 4. Finally, among all the extensions of the theory, we have selected two of them for reviewing: the case of higher-order autonomous dynamical systems (Lagrangian and Hamiltonian formalisms), which is a direct application of the above general framework, and the generalization to the Lagrangian and Hamiltonian multisymplectic formalisms of first-order classical field theories, which can also be interpreted as a special case of the general framework. Both of them are treated in Section 5.

Throughout the work it is considered that the manifolds are real, smooth, and second countable. In the same way, all the maps are assumed to be smooth. The summation convention for repeated cross indices is also assumed.

\section{The Geometric Hamilton-Jacobi Theory}

We summarize the main features of the geometric Hamilton-Jacobi theory for the Hamiltonian and Lagrangian formalisms of autonomous dynamical systems, as it is stated in Reference [14] (also see Reference [9,15]).

\subsection{Hamiltonian Hamilton-Jacobi Problem}

Typically, a (regular autonomous) Hamiltonian system is a triad $\left(\mathrm{T}^{*} Q, \omega, H\right)$, where the bundle $\pi_{Q}: T^{*} Q \rightarrow Q$ represents the phase space of a dynamical system ( $Q$ is the configuration space), $\omega=-\mathrm{d} \theta \in \Omega^{2}\left(\mathrm{~T}^{*} Q\right)$ is the natural symplectic form in $\mathrm{T}^{*} Q$, and $H \in \mathrm{C}^{\infty}\left(\mathrm{T}^{*} Q\right)$ is the Hamiltonian function. The dynamical trajectories are the integral curves $\sigma: I \subseteq \mathbb{R} \longrightarrow \mathrm{T}^{*} Q$ of the Hamiltonian vector field $Z_{H} \in \mathfrak{X}\left(\mathrm{T}^{*} Q\right)$ associated with $H$, which is the solution to the Hamiltonian equation

$$
i\left(Z_{H}\right) \omega=\mathrm{d} H .
$$


(Here, $\Omega^{k}\left(\mathrm{~T}^{*} Q\right)$ and $\mathfrak{X}\left(\mathrm{T}^{*} Q\right)$ are the sets of differentiable $k$-forms and vector fields in $T^{*} Q$, and $i\left(Z_{H}\right) \omega$ denotes the inner contraction of $Z_{H}$ and $\omega$ ). In natural coordinates $\left(q^{i}, p_{i}\right)$ of $\mathrm{T}^{*} Q$, we have that $\omega=\mathrm{d} q^{i} \wedge \mathrm{d} p_{i}$, and the curves $\sigma(t)=\left(q^{i}(t), p_{i}(t)\right)$ are the solution to the Hamilton equations

$$
\frac{\mathrm{d} q^{i}}{\mathrm{~d} t}=\frac{\partial H}{\partial p_{i}}(q(t), p(t)), \quad \frac{\mathrm{d} p_{i}}{\mathrm{~d} t}=-\frac{\partial H}{\partial q^{i}}(q(t), p(t)) .
$$

Definition 1. The generalized Hamiltonian Hamilton-Jacobi problem for a Hamiltonian system $\left(\mathrm{T}^{*} Q, \omega, H\right)$ is to find a vector field $X \in \mathfrak{X}(Q)$ and a 1-form $\alpha \in \Omega^{1}(Q)$ such that, if $\gamma: \mathbb{R} \rightarrow Q$ is an integral curve of $X$, then $\alpha \circ \gamma: \mathbb{R} \rightarrow \mathrm{T}^{*} Q$ is an integral curve of $Z_{H}$, i.e., if $X \circ \gamma=\dot{\gamma}$, then $\overline{\alpha \circ \gamma}=Z_{H} \circ(\alpha \circ \gamma)$. Then, the couple $(X, \alpha)$ is a solution to the generalized Hamiltonian Hamilton-Jacobi problem.

Theorem 1. The following statements are equivalent:

1. The couple $(X, \alpha)$ is a solution to the generalized Hamiltonian Hamilton-Jacobi problem.

2. The vector fields $X$ and $Z_{H}$ are $\alpha$-related, i.e., $Z_{H} \circ \alpha=T \alpha \circ X$. As a consequence, $X=$ $\mathrm{T} \pi_{Q} \circ Z_{H} \circ \alpha$, and it is called the vector field associated with the form $\alpha$.

3. The submanifold $\operatorname{Im} \alpha$ of $\mathrm{T}^{*} Q$ is invariant by the Hamiltonian vector field $Z_{H}$ (or, which means the same thing, $Z_{H}$ is tangent to $\operatorname{Im} \alpha$ ).

4. The integral curves of $Z_{H}$ which have their initial conditions in $\operatorname{Im} \alpha$ project onto the integral curves of $X$.

5. The equation $i(X) \mathrm{d} \alpha=-\mathrm{d}\left(\alpha^{*} H\right)$ holds for the 1-form $\alpha$.

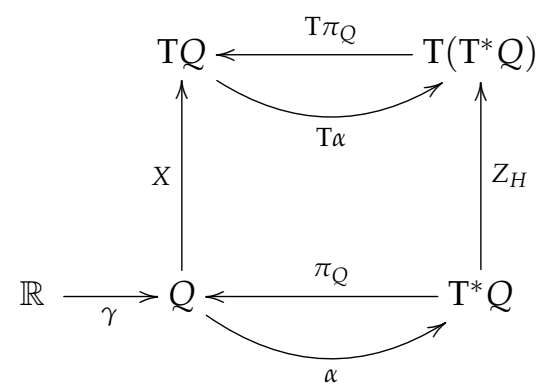

Proof. (Guidelines for the proof):

The equivalence between 1 and 2 is a consequence of the Definition 1 and the definition of integral curves. Then, the expression $X=\mathrm{T} \pi_{Q} \circ Z_{H} \circ \alpha$ is obtained by composing both members of the equality $Z_{H} \circ \alpha=\mathrm{T} \alpha \circ X$ with $\mathrm{T} \pi_{Q}$ and taking into account that $\pi_{Q} \circ \alpha=\operatorname{Id}_{Q}$.

Items 3 and 4 follow from 2 .

Item 5 is obtained from Definition 1 and using the dynamical Equation (1).

In order to solve the generalized Hamilton-Jacobi problem, it is usual to state a less general version of it, which constitutes the standard Hamilton-Jacobi problem.

Definition 2. The Hamiltonian Hamilton-Jacobi problem for a Hamiltonian system $\left(\mathrm{T}^{*} Q, \omega, H\right)$ is to find a 1-form $\alpha \in \Omega^{1}(Q)$ such that it is a solution to the generalized Hamiltonian Hamilton-Jacobi problem and is closed. Then, the form $\alpha$ is a solution to the Hamiltonian Hamilton-Jacobi problem.

As $\alpha$ is closed, for every point in $Q$, there is a function $S$ in a neighborhood $U \subset Q$ such that $\alpha=\mathrm{d} S$. It is called a local generating function of the solution $\alpha$.

Theorem 2. The following statements are equivalent:

1. The form $\alpha \in \Omega^{1}(Q)$ is a solution to the Hamiltonian Hamilton-Jacobi problem. 
2. $\operatorname{Im} \alpha$ is a Lagrangian submanifold of $\mathrm{T}^{*} Q$ which is invariant by $Z_{H}$, and $S$ is a local generating function of this Lagrangian submanifold.

3. The equation $\mathrm{d}\left(\alpha^{*} H\right)=0$ holds for $\alpha$, or, which is equivalent, the function $H \circ \mathrm{d} S: Q \rightarrow \mathbb{R}$ is locally constant.

Proof. (Guidelines for the proof): They are consequences of Theorem 1 and Definition 2.

The last condition, written in natural coordinates, gives the classical form of the Hamiltonian Hamilton-Jacobi equation, which is

$$
H\left(q^{i}, \frac{\partial S}{\partial q^{i}}\right)=E(c t n .) .
$$

These forms $\alpha$ are particular solutions to the (generalized) Hamilton-Jacobi problem, but we are also interested in finding complete solutions to the problem. Then,

Definition 3. Let $\Lambda \subseteq \mathbb{R}^{n}$. A family of solutions $\left\{\alpha_{\lambda} ; \lambda \in \Lambda\right\}$, depending on $n$ parameters $\lambda \equiv\left(\lambda_{1}, \ldots, \lambda_{n}\right) \in \Lambda$, is a complete solution to the Hamiltonian Hamilton-Jacobi problem if the map

$$
\begin{aligned}
& \Phi: Q \times \Lambda \quad \longrightarrow \quad \mathrm{T}^{*} \mathrm{Q} \\
& (q, \lambda) \mapsto \alpha_{\lambda}(q)
\end{aligned}
$$

is a local diffeomorphism.

Remark 1. Given a complete solution $\left\{\alpha_{\lambda} ; \lambda \in \Lambda\right\}$, as $\mathrm{d} \alpha_{\lambda}=0, \forall \lambda \in \Lambda$, there is a family of functions $\left\{S_{\lambda}\right\}$ defined in neighborhoods $U_{\lambda} \subset Q$ of every point such that $\alpha_{\lambda}=\mathrm{d} S_{\lambda}$. Therefore, we have a locally defined function

$$
\begin{aligned}
& \mathcal{S}: \cap U_{\lambda} \times \Lambda \subset Q \times \Lambda \quad \longrightarrow \quad \mathbb{R} \\
& (q, \lambda) \quad \mapsto S_{\lambda}(q)^{\prime}
\end{aligned}
$$

which is called a local generating function of the complete solution $\left\{\alpha_{\lambda} ; \lambda \in \Lambda\right\}$.

A complete solution defines a Lagrangian foliation in $\mathrm{T}^{*} Q$ which is transverse to the fibers, and such that $Z_{H}$ is tangent to the leaves. The functions that locally define this foliation are the components of a map $F: \mathrm{T}^{*} Q \stackrel{\Phi^{-1}}{\longrightarrow} Q \times \Lambda \stackrel{\mathrm{pr}_{2}}{\longrightarrow} \Lambda \subset \mathbb{R}^{n}$ and give a family of constants of motion of $Z_{H}$. Conversely, if we have $n$ first integrals $f_{1}, \ldots, f_{n}$ of $Z_{H}$ in involution, such that $\mathrm{d} f_{1} \wedge \ldots \wedge \mathrm{d} f_{n} \neq 0$, then $f_{i}=\lambda_{i}$, with $\lambda_{i} \in \mathbb{R}$, define this transversal Lagrangian foliation, hence having a local complete solution $\left\{\alpha_{\lambda}, \lambda \in \Lambda\right\}$. Thus, we can locally isolate $p_{i}=p_{i}(q, \lambda)$, replace them in $Z_{H}$, and project to the basis, then obtaining the family of vector fields $\left\{X_{\lambda}\right\}$ associated with the local complete solution. If $\left\{\alpha_{\lambda} ; \lambda \in \Lambda\right\}$ is a complete solution, then all the integral curves of $Z_{H}$ are obtained from the integral curves of the associated vector fields $\left\{X_{\lambda}\right\}$.

\subsection{Lagrangian Hamilton-Jacobi Problem}

The above framework for the Hamilton-Jacobi theory can be easily translated to the Lagrangian formalism of mechanics. Now, the phase space is the tangent bundle $\tau_{Q}: T Q \rightarrow Q$ of the configuration bundle $Q$ and the dynamics is given by the Lagrangian function of the system, $\mathcal{L} \in \mathrm{C}^{\infty}(\mathrm{T} Q)$. Using the canonical structures in $\mathrm{T} Q$, i.e., the vertical endomorphism $S \in \mathcal{T}_{1}^{1}(\mathrm{TQ})$, and the Liouville vector field $\Delta \in \mathfrak{X}(\mathrm{TQ})$, the Lagrangian forms $\theta_{\mathcal{L}}:=\mathrm{d} \mathcal{L} \circ S \in \Omega^{1}(\mathrm{~T} Q), \omega_{\mathcal{L}}=-d \theta_{\mathcal{L}} \in \Omega^{2}(\mathrm{~T} Q)$, and the Lagrangian energy $E_{\mathcal{L}}:=\Delta(\mathcal{L})-\mathcal{L} \in \mathrm{C}^{\infty}(\mathrm{T} Q)$ are constructed. Then, the Lagrangian equation is

$$
i\left(\Gamma_{\mathcal{L}}\right) \omega_{\mathcal{L}}=\mathrm{d} E_{\mathcal{L}}
$$

and $\left(\mathrm{TQ}, \omega_{\mathcal{L}}, E_{\mathcal{L}}\right)$ is a Lagrangian dynamical system. Furthermore, the Legendre transformation associated with $\mathcal{L}$, denoted by $\mathcal{F} \mathcal{L}: \mathrm{T} Q \rightarrow \mathrm{T}^{*} Q$, is defined as the fiber derivative of the Lagrangian function. We assume that $\mathcal{L}$ is regular, i.e., $\mathcal{F} \mathcal{L}$ is a local diffeomor- 
phism, or, equivalently, $\omega_{\mathcal{L}}$ is a symplectic form (the Lagrangian is hyper-regular if $\mathcal{F} \mathcal{L}$ is a global diffeomorphism). In that case, the Lagrangian Equation (3) has a unique solution $\Gamma_{\mathcal{L}} \in \mathfrak{X}(\mathrm{TQ})$, which is called the Lagrangian vector field, in which integral curves are holonomic, and are the solutions to the Euler-Lagrange equations. (see Reference [49] for details).

Definition 4. The generalized Lagrangian Hamilton-Jacobi problem for a Lagrangian system $\left(\mathrm{TQ}, \omega_{\mathcal{L}}, E_{\mathcal{L}}\right)$ is to find a vector field $X \in \mathfrak{X}(Q)$ such that, if $\gamma: \mathbb{R} \rightarrow Q$ is an integral curve of $X$, then $X \circ \gamma=\dot{\gamma}: \mathbb{R} \rightarrow \mathrm{T} Q$ is an integral curve of $\Gamma_{\mathcal{L}}$, i.e., if $X \circ \gamma=\dot{\gamma}$, then $\Gamma_{\mathcal{L}} \circ \dot{\gamma}=\overline{X \circ \gamma}$. Then, the vector field $X$ is a solution to the generalized Lagrangian Hamilton-Jacobi problem.

Theorem 3. The following statements are equivalent:

1. The vector field $X$ is a solution to the generalized Lagrangian Hamilton-Jacobi problem.

2. The vector fields $X$ and $\Gamma_{\mathcal{L}}$ are $X$-related, i.e., $\Gamma_{\mathcal{L}} \circ X=T X \circ X$.

3. The submanifold $\operatorname{Im} X$ of $\mathrm{T} Q$ is invariant by the Lagrangian vector field $\Gamma_{\mathcal{L}}$ (or, which means the same thing, $\Gamma_{\mathcal{L}}$ is tangent to $\left.\operatorname{Im} X\right)$.

4. The integral curves of $\Gamma_{\mathcal{L}}$ which have their initial conditions in $\operatorname{Im} X$ project onto the integral curves of $X$.

5. The equation $i(X)\left(X^{*} \omega_{\mathcal{L}}\right)=\mathrm{d}\left(X^{*} E_{\mathcal{L}}\right)$ holds for the vector field $X$.

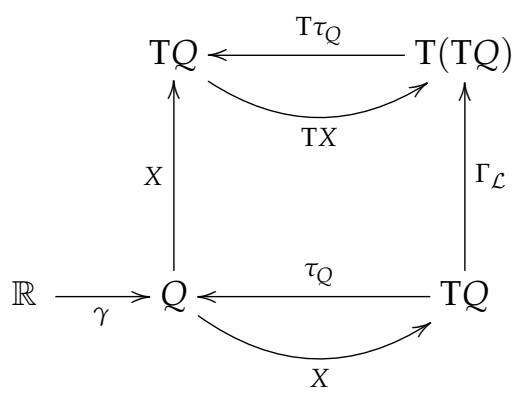

Proof. (Guidelines for the proof): The proof follows the same patterns as Theorem 1.

As in the Hamiltonian formalism, we consider the following simpler case:

Definition 5. The Lagrangian Hamilton-Jacobi problem for a Lagrangian system $\left(\mathrm{TQ}, \omega_{\mathcal{L}}, E_{\mathcal{L}}\right)$ is to find a vector field $X$ such that it is a solution to the generalized Lagrangian Hamilton-Jacobi problem and satisfies that $X^{*} \omega_{\mathcal{L}}=0$. Then, this vector field $X$ is a solution to the Lagrangian Hamilton-Jacobi problem.

Since $0=X^{*} \omega_{\mathcal{L}}=-X^{*} \mathrm{~d} \theta_{\mathcal{L}}=-d\left(X^{*} \theta_{\mathcal{L}}\right)$, then, for every point of $Q$, there is a neighborhood $U \subset Q$ and a function $S$ such that $X^{*} \theta_{\mathcal{L}}=\mathrm{d} S$ (in $U$ ).

Theorem 4. The following statements are equivalent:

1. The vector field $X$ is a solution to the Lagrangian Hamilton-Jacobi problem.

2. Im $X$ is a Lagrangian submanifold of $\mathrm{TQ}$ which is invariant by the Lagrangian vector field $\Gamma_{\mathcal{L}}$ (and $S$ is a local generating function of this Lagrangian submanifold).

3. The equation $\mathrm{d}\left(X^{*} E_{\mathcal{L}}\right)=0$ holds for $X$, or, which is equivalent, the function $E_{\mathcal{L}} \circ \mathrm{d} S: Q \rightarrow \mathbb{R}$ is locally constant.

Proof. (Guidelines for the proof): They are consequences of Theorem 3 and Definition 5. 
The last condition leads to the following expression which is the form of the Lagrangian Hamilton-Jacobi equation in natural coordinates,

$$
\frac{\partial S}{\partial q^{i}}=\frac{\partial \mathcal{L}}{\partial v^{i}}\left(q^{i}, X^{i}\right) .
$$

As in the Hamiltonian Hamilton-Jacobi theory, we are interested in the complete solutions to the problem, which are defined as:

Definition 6. Let $\Lambda \subseteq \mathbb{R}^{n}$. A family of solutions $\left\{X_{\lambda} ; \lambda \in \Lambda\right\}$, depending on $n$ parameters $\lambda \equiv\left(\lambda_{1}, \ldots, \lambda_{n}\right) \in \Lambda$, is a complete solution to the Lagrangian Hamilton-Jacobi problem if the map

$$
\begin{aligned}
& \Psi: Q \times \Lambda \longrightarrow \mathrm{TQ} \\
& (q, \lambda) \mapsto X_{\lambda}(q)
\end{aligned}
$$

is a local diffeomorphism.

If we have a complete solution to the Lagrangian Hamilton-Jacobi problem, all the integral curves of the Lagrangian vector field $\Gamma_{\mathcal{L}}$ are obtained from the integral curves of all the vector fields $X_{\lambda}$.

The equivalence between the Lagrangian and the Hamiltonian Hamilton-Jacobi problems is stated as follows:

Theorem 5. Let $\left(\mathrm{T} Q, \omega_{\mathcal{L}}, E_{\mathcal{L}}\right)$ be a (hyper)regular Lagrangian system, and $\left(\mathrm{T}^{*} Q, \omega, H\right)$ its associated Hamiltonian system. If $\alpha \in \Omega^{1}(Q)$ is a solution to the (generalized) Hamiltonian HamiltonJacobi problem, then $X=\mathcal{F} \mathcal{L}^{-1} \circ \alpha$ is a solution to the (generalized) Lagrangian Hamilton-Jacobi problem; conversely, if $X \in \mathfrak{X}(Q)$ is a solution to the (generalized) Lagrangian Hamilton-Jacobi problem, then $\alpha=\mathcal{F} \mathcal{L} \circ X$ is a solution to the (generalized) Hamiltonian Hamilton-Jacobi problem.

Proof. (Guidelines for the proof): It can be proven that $\alpha=\mathcal{F} \mathcal{L} \circ X$; then, bearing in mind that $\mathrm{T} \mathcal{F} \mathcal{L} \circ \Gamma_{\mathcal{L}}=Z_{H} \circ \mathcal{F} \mathcal{L}$, the proof follows using items 2 and 5 of Theorems 1 and 3 (or item 3 of Theorems 2 and 4 ).

\section{The "Classical" Hamilton-Jacobi Theory}

In this section, we review the geometric description of the classical Hamiltonian Hamilton-Jacobi theory (for autonomous systems), based on using canonical transformations $[1,2,9,12,13]$. It is stated in the Hamiltonian formalism.

\subsection{Canonical Transformations and the Classical Hamiltonian Hamilton-Jacobi Problem}

First, we recall the following well-known results [9]:

Proposition 1. Let $\left(M_{1}, \omega_{1}\right),\left(M_{2}, \omega_{2}\right)$ be symplectic manifolds and $\pi_{j}: M_{1} \times M_{2} \rightarrow M_{j}$, $j=1$, 2. Then, $\left(M_{1} \times M_{2}, \pi_{1}^{*} \omega_{1}-\pi_{2}^{*} \omega_{2}\right)$ is a symplectic manifold.

Proposition 2. Let $\Phi: M_{1} \longrightarrow M_{2}$ be a diffeomorphism and $\mathrm{j}$ : graph $\Phi \hookrightarrow M_{1} \times M_{2}$. $\Phi$ is a symplectomorphism (i.e., $\Phi^{*} \omega_{2}=\omega_{1}$ ) if, and only if, graph $\Phi$ is a Lagrangian submanifold of $\left(M_{1} \times M_{2}, \pi_{1}^{*} \omega_{1}-\pi_{2}^{*} \omega_{2}\right)$.

$$
\begin{aligned}
& \text { If } \omega_{j}=-\mathrm{d} \theta_{j}, j=1,2 \text {; being graph } \Phi \text { a Lagrangian submanifold, we have } \\
& \qquad \begin{array}{l}
0 \jmath^{*}\left(\pi_{1}^{*} \omega_{1}-\pi_{2}^{*} \omega_{2}\right)=\left.\mathrm{d} \jmath^{*}\left(\pi_{2}^{*} \theta_{2}-\pi_{1}^{*} \theta_{1}\right) \Longleftrightarrow \jmath^{*}\left(\pi_{2}^{*} \theta_{2}-\pi_{1}^{*} \theta_{1}\right)\right|_{\mathcal{W}}=-\mathrm{d} \mathcal{S} .
\end{array}
\end{aligned}
$$

$\mathcal{S}$ is a function defined in an open neighborhood $\mathcal{W} \subset \operatorname{graph} \Phi$ of every point, which depends on the choice of $\theta_{1}$ and $\theta_{2}$. 
Definition 7. $\mathcal{S}$ is called a generating function of the Lagrangian submanifold graph $\Phi$ and, hence, of the symplectomorphism $\Phi$.

If $\left(\mathcal{U}_{1} ; q^{i}, p_{i}\right),\left(\mathcal{U}_{2} ; \tilde{q}^{i}, \tilde{p}_{i}\right)$ are Darboux charts such that $\mathcal{W} \subset \mathcal{U}_{1} \times \mathcal{U}_{2}$, local coordinates in $\mathcal{W}$ can be chosen in several ways. This leads to different possible choices for $\mathcal{S}$. Thus, for instance, if $\left(\mathcal{W} ; q^{i} \tilde{q}^{i}\right)$ is a chart, then (5) gives the symplectomorphism explicitly as

$$
\tilde{p}_{i} \mathrm{~d} \tilde{q}^{i}-p_{i} \mathrm{~d} q^{i}=-\mathrm{d} \mathcal{S}(q, \tilde{q}) \quad \Longleftrightarrow \quad \tilde{p}_{i}=-\frac{\partial \mathcal{S}}{\partial \tilde{q}^{i}}(q, \tilde{q}), p_{i}=\frac{\partial \mathcal{S}}{\partial q^{i}}(q, \tilde{q}) .
$$

Now, let $\left(T^{*} Q, \omega, H\right)$ be a Hamiltonian system.

Definition 8. A canonical transformation for a Hamiltonian system $\left(\mathrm{T}^{*} Q, \omega, H\right)$ is a symplectomorphism $\Phi: \mathrm{T}^{*} Q \rightarrow \mathrm{T}^{*} Q$. As a consequence, $\Phi$ transforms Hamiltonian vector fields into Hamiltonian vector fields.

Definition 9. The Hamilton-Jacobi problem for a Hamiltonian system $\left(T^{*} Q, \omega, H\right)$ is to find a canonical transformation $\Phi: \mathrm{T}^{*} Q \rightarrow \mathrm{T}^{*} Q$ leading the system to equilibrium, i.e., such that $H \circ \Phi=E(c t n$.).

The canonical transformation $\Psi$ is given by a generating function $\mathcal{S}$ :

$$
\frac{\partial \mathcal{S}}{\partial q^{i}}(q, \tilde{q})=p_{i} \quad, \quad-\frac{\partial \mathcal{S}}{\partial \tilde{q}^{i}}(q, \tilde{q})=\tilde{p}_{i},
$$

where $\mathcal{S}$ the general solution to the Hamilton-Jacobi equation

$$
H\left(q^{i}, \frac{\partial \mathcal{S}}{\partial q^{i}}\right)=E(\operatorname{ctn} .) .
$$

Then, the Hamilton equations for the transformed Hamiltonian function $H \circ \Phi \equiv$ $\tilde{H}$ are

$$
\frac{\mathrm{d} \tilde{q}^{i}}{\mathrm{~d} t}=\frac{\partial \tilde{H}}{\partial \tilde{p}_{i}}(\tilde{q}(t), \tilde{p}(t))=0 \quad, \quad \frac{\mathrm{d} \tilde{p}_{i}}{\mathrm{~d} t}=-\frac{\partial \tilde{H}}{\partial \tilde{q}^{i}}(\tilde{q}(t), \tilde{p}(t))=0 ;
$$

and solving (7), from (8) and (6), the dynamical curves $\left(q^{i}(t), p_{i}(t)\right)$ of the original Hamiltonian system $\left(\mathrm{T}^{*} Q, \omega, H\right)$ are obtained.

\subsection{Relation between the "Classical" and the Geometric Hamilton-Jacobi Theories}

The relation between the "classical" and the geometric Hamilton-Jacobi theories is established through the equivalence of complete solutions and canonical transformations (also see Reference [48]).

Theorem 6. Let $\left(\mathrm{T}^{*} Q, \omega, H\right)$ be a Hamiltonian system. A complete solution $\left\{\alpha_{\lambda} ; \lambda \in \Lambda\right\}$ to the Hamilton-Jacobi problem provides a canonical transformation $\Phi: \mathrm{T}^{*} Q \rightarrow \mathrm{T}^{*} Q$ leading the system to equilibrium, and conversely.

Proof. In a neighborhood of every point, consider a complete solution $\left\{\alpha_{\lambda} ; \lambda \in \Lambda\right\}$, and let $\mathcal{S}$ be a generating function of it. As $\mathcal{S}=\mathcal{S}\left(q^{i}, \lambda^{i}\right)$, we can identify $\lambda^{i}$ with a subset of coordinates $\lambda^{i} \equiv \tilde{q}^{i}$ in $\mathrm{T}^{*} Q \times \mathrm{T}^{*} Q$, and then $\mathcal{S}=\mathcal{S}\left(q^{i}, \tilde{q}^{i}\right)$ can be thought as a generating function of a local canonical transformation $\Phi$ and, hence, of an open set $\mathcal{W}$ of the Lagrangian submanifold graph $\Phi \hookrightarrow \mathrm{T}^{*} Q \times \mathrm{T}^{*} Q$. Making this construction in every chart, we have the transformation $\Phi$ and the submanifold graph $\Psi$. Now, as (2) holds for every particular solution $S_{\lambda}$, we have that

$$
E=H\left(q^{i}(\tilde{q}, \tilde{p}), \frac{\partial \mathcal{S}}{\partial q^{i}}(q(\tilde{q}, \tilde{p}), \tilde{q})\right)=\tilde{H}\left(\tilde{q}^{i}, \tilde{p}_{i}\right) .
$$


Conversely, if we have the canonical transformation $\Psi$, from a generating function $\mathcal{S}=$ $\mathcal{S}\left(q^{i}, \tilde{q}^{i}\right)$, taking $\tilde{q} \equiv\left(\tilde{q}^{i}\right)=\left(\lambda^{i}\right) \equiv \lambda$, we obtain a family of functions $\left\{S_{\lambda}\right\}$ and, hence, a local complete solution $\left\{\alpha_{\lambda}=\mathrm{d} S_{\lambda} ; \lambda \in \Lambda\right\}$ to the Hamiltonian Hamilton-Jacobi problem. Making this construction in every chart, we have the complete solution.

Geometrically, this means that, on each local chart of $T^{*} Q$, fixing the coordinates $\tilde{q}^{i}=\lambda^{i}$ of a point, we obtain a local submanifold wherein the image by $\Phi^{-1}$ gives the image of a local section $\alpha_{\lambda}: Q \rightarrow \mathrm{T}^{*} Q$ which is a particular solution to the Hamiltonian Hamilton-Jacobi problem.

\section{General Geometric Framework for the Hamilton-Jacobi Theory}

The geometric Hamilton-Jacobi theory can be stated in a more general framework which allows us to extend the theory to a wide variety of systems and situations. Next, we present a summary of this general framework, as it is stated in Reference [36] (also see Reference [31] for another similar approach).

\subsection{Slicing Problems}

In general, a dynamical system is just a couple $(P, Z)$, where $P$ is a manifold and $Z \in \mathfrak{X}(P)$ is a vector field which defines the dynamical equation on $P$. Then, in order to state the analogous to the Hamilton-Jacobi problem for this system in a more general context, consider a manifold $\mathcal{M}$, a vector field $X \in \mathfrak{X}(\mathcal{M})$, and a map $\alpha: \mathcal{M} \rightarrow P$, as it is showed in the following diagram:

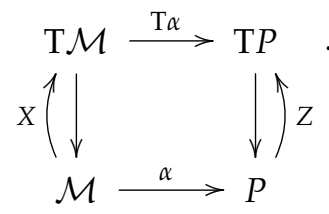

Proposition 3. The following statements are equivalent:

1. If $\gamma$ is an integral curve of $X$, then $\zeta=\alpha \circ \gamma$ is an integral curve of $Z$.

2. The vector fields $X$ and $Z$ are $\alpha$-related:

$$
\mathrm{T} \alpha \circ X=\mathrm{Z} \circ \alpha .
$$

Furthermore, if $\alpha$ is an injective immersion, (inducing a diffeomorphism $\alpha_{0}: \mathcal{M} \rightarrow \alpha(\mathcal{M})$ ), then these properties are equivalent to:

3. The vector field $Z$ is tangent to $\alpha(\mathcal{M})$, and, if $Z_{o}=\left.Z\right|_{\alpha(\mathcal{M})}$, then $X=\alpha_{o}^{*}\left(Z_{o}\right)$.

Then, the map $\gamma \mapsto \alpha \circ \xi$ is a bijection between the integral curves of $X$ and the integral curves of $Z$ in $\alpha(\mathcal{M})$.

Proof. They are immediate, bearing in mind the commutativity of the above diagram.

Definition 10. A slicing of a dynamical system $(P, Z)$ is a triple $(\mathcal{M}, \alpha, X)$ which is a solution to the slicing Equation (9).

If $\left(x^{i}\right)$ and $\left(z^{j}\right)$ are coordinates in $\mathcal{M}$ and $P$, respectively, and $\alpha(x)=\left(a^{j}(x)\right), X=$ $X^{i} \frac{\partial}{\partial x^{i}}$, and $Z=Z^{j} \frac{\partial}{\partial z^{j}}$, then $(T \alpha \circ X-Z \circ \alpha)\left(x^{i}\right)=\left(a^{j}(x), \frac{\partial a^{j}}{\partial x^{i}} X^{i}-Z^{j}(\alpha(x))\right)$, and $(\mathcal{M}, \alpha, X)$ is a solution to the slicing equation if, and only if,

$$
\frac{\partial a^{j}}{\partial x^{i}} X^{i}(x)=Z^{j}(\alpha(x))
$$

We say that the vector field $X$ gives a "partial dynamics" or a "slice" of the "whole dynamics" which is given by $Z$, and the whole dynamics can be recovered from these slices. 
In fact, the integral curves of $Z$ contained in $\alpha(\mathcal{M}) \subset P$ can be described by a solution $(\alpha, X)$ to the slicing equation; but we need a complete solution to describe all the integral curves of $Z$, and it can be defined as a family of solutions depending on the parameters of a space $\Lambda \subseteq \mathbb{R}^{n}$.

Definition 11. A complete slicing of a dynamical system $(P, Z)$ is a map $\bar{\alpha}: \mathcal{M} \times \Lambda \rightarrow P$ and a vector field $\bar{X}: \mathcal{M} \times \Lambda \longrightarrow \mathrm{T} \mathcal{M}$ along the projection $\mathcal{M} \times \Lambda \longrightarrow \mathcal{M}$ such that:

1. The map $\bar{\alpha}$ is surjective,

2. for every $\lambda \in \Lambda$, the map $\alpha_{\lambda}: \mathcal{M} \rightarrow P$ and $X_{\lambda}: \mathcal{M} \rightarrow T \mathcal{M}$ are a slicing of $Z$.

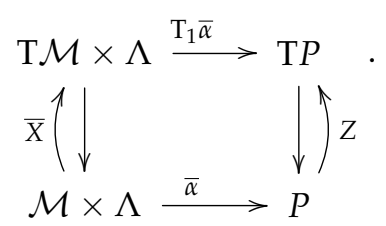

Thus, a complete slicing is a family of maps $\alpha_{\lambda} \equiv \bar{\alpha}(\cdot, \lambda): \mathcal{M} \rightarrow P$ and vector fields $X_{\lambda} \equiv \bar{X}(\cdot, \lambda): \mathcal{M} \rightarrow \mathrm{T} \mathcal{M}$ satisfying the above conditions.

As for every point $p \in P$ there exists $(x, \lambda) \in \mathcal{M} \times \Lambda$ such that $\bar{\alpha}(x, \lambda)=p$, the integral curve of $Z$ through $p$ is described by the integral curve of $X_{\lambda}$ through $x$ by means of the map $\alpha_{\lambda}$. In addition, if each $\alpha_{\lambda}$ is an immersion (for instance, when it is a diffeomorphism), then $X_{\lambda}$ are determined by the $\alpha_{\lambda}$.

The hypothesis of $\alpha$ being an embedding holds in many situations, for instance, for the sections of a fiber bundle $\pi: P \longrightarrow \mathcal{M}$. Then, we can consider the slicing problem for sections $\alpha: \mathcal{M} \rightarrow P$ of $\pi$, as before. In this case, as $\alpha$ is an embedding, Equation (9) determines $X$, and $X$ is given from $\alpha$ by the equation

$$
X=\mathrm{T} \pi \circ \mathrm{Z} \circ \alpha .
$$

In this case, Proposition 3 states that a section $\alpha$ of $\pi: P \rightarrow \mathcal{M}$ is a solution to the slicing equation for $(P, Z)$ if, and only if,

$$
\mathrm{T} \alpha \circ \mathrm{T} \pi \circ \mathrm{Z} \circ \alpha=\mathrm{Z} \circ \alpha .
$$

4.2. Recovering the Hamilton-Jacobi Equation for Hamiltonian and Lagrangian Dynamical Systems

Consider the case of a Hamiltonian system $(P, \omega, H)$, where $(P, \omega)$ is a symplectic manifold, $H \in C^{\infty}(P)$ is a Hamiltonian function, and $Z=Z_{H}$ is its Hamiltonian vector field, i.e., the solution to (1). Then,

Theorem 7. If $(\mathcal{M}, \alpha, X)$ is a solution to the slicing equation (9) for $\left(P, Z_{H}\right)$, then

$$
i(X) \alpha^{*} \omega-\mathrm{d} \alpha^{*} H=0 .
$$

In addition, if $\alpha: \mathcal{M} \rightarrow P$ is an embedding satisfying the condition $\alpha^{*} \omega=0$, then

$$
\mathrm{d}\left(\alpha^{*} H\right)=0 ；
$$

conversely, if $\operatorname{dim} P=2 \operatorname{dim} \mathcal{M}$ and $\alpha$ satisfies this equation and $\alpha^{*} \omega=0$, then $\alpha$ is a solution to the slicing Equation (9). 
In the particular case where $\pi: P \rightarrow \mathcal{M}$ is a fiber bundle (for instance, $\mathcal{M}=Q$ and $\left.P=\mathrm{T}^{*} Q\right)$, we can consider the slicing problem as before, but only for sections of $\pi$,

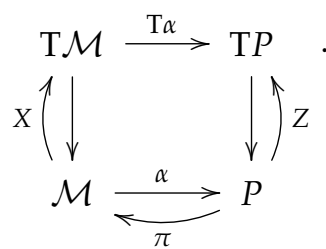

Being $\alpha$ an embedding, the Equation (9) determines $X$, and, composing this equation with $\mathrm{T} \pi$, we obtain that $X=\mathrm{T} \pi \circ Z_{H} \circ \alpha$. Therefore, the slicing Equation (9) reads

$$
\mathrm{T} \alpha \circ \mathrm{T} \pi \circ \mathrm{Z} \circ \alpha=\mathrm{Z} \circ \alpha
$$

In this way, Equation (9) can be considered as a generalization of the Hamilton-Jacobi equation in the Hamiltonian formalism, which is just the slicing equation for a closed 1 -form $\alpha$ in $Q$. Therefore, $\alpha=\mathrm{d} S$ locally, and the slicing equation looks in the ordinary form $H \circ \mathrm{d} S=$ const.

The same applies to the Lagrangian formalism. In this case $P=\mathrm{T} Q$, and, if $\mathcal{L} \in$ $\mathrm{C}^{\infty}(\mathrm{T} Q)$ is a regular Lagrangian function, $Z=\Gamma_{\mathcal{L}}$ is the Lagrangian vector field solution to the Lagrangian Equation (3). Then, all proceeds as in the Hamiltonian case.

The Definitions 3 and 6 of complete solutions to the Hamiltonian and Lagrangian Hamilton-Jacobi problems, respectively, are particular cases of the Definition 11 of complete slicings.

\section{The Hamilton-Jacobi Problem for Other Physical Systems}

Using the general framework presented in the above section, the Hamilton-Jacobi problem can be stated for a wide kind of physical systems. Next, we review two of them. Other applications of the theory are listed in detail in the Introduction.

\subsection{Higher-Order (Autonomous) Dynamical Systems}

Let $Q$ be a $n$-dimensional manifold and let $\mathrm{T}^{k} Q$ the $k$ th-order tangent bundle of $Q$, which is endowed with natural coordinates $\left(q_{0}^{A}, q_{1}^{A}, \ldots, q_{k}^{A}\right)=\left(q_{i}^{A}\right), 0 \leq i \leq k, 1 \leq A \leq n$. If $\mathcal{L} \in \mathrm{C}^{\infty}\left(\mathrm{T}^{k} Q\right)$ is the Lagrangian function of an autonomous $k$ th-order Lagrangian system, using the canonical structures of the higher-order tangent bundles, we can construct the Poincaré-Cartan forms and the Lagrangian energy in which coordinate expressions are

$$
\begin{aligned}
& \omega_{\mathcal{L}}=-\mathrm{d} \theta_{\mathcal{L}}=\sum_{r=1}^{k} \sum_{i=0}^{k-r}(-1)^{i+1} d_{T}^{i} \mathrm{~d}\left(\frac{\partial \mathcal{L}}{\partial q_{r+i}^{A}}\right) \wedge \mathrm{d} q_{r-1}^{A} \in \Omega^{2}\left(\mathrm{~T}^{2 k-1} Q\right), \\
& E_{\mathcal{L}}=\sum_{r=1}^{k} q_{r}^{A} \sum_{i=0}^{k-r}(-1)^{i} d_{T}^{i}\left(\frac{\partial \mathcal{L}}{\partial q_{r+i}^{A}}\right)-\mathcal{L} \in \mathrm{C}^{\infty}\left(\mathrm{T}^{2 k-1} Q\right),
\end{aligned}
$$

where $d_{T} f\left(q_{0}^{A}, \ldots, q_{k+1}^{A}\right)=\sum_{i=0}^{k} q_{i+1}^{A} \frac{\partial f}{\partial q_{i}^{A}}\left(q_{0}^{A}, \ldots, q_{k}^{A}\right)$, and $d^{i}=d \circ \stackrel{(i)}{\ldots} \circ{ }^{\circ}$. Thus, we have the higher-order Lagrangian system $\left(\mathrm{T}^{2 k-1} Q, \omega_{\mathcal{L}}, E_{\mathcal{L}}\right)$. Assuming that the Lagrangian function is regular, i.e., $\omega_{\mathcal{L}}$ is a symplectic form, the Lagrangian equation $i\left(X_{\mathcal{L}}\right) \omega_{\mathcal{L}}=$ $\mathrm{d} E_{\mathcal{L}}$ has a unique solution $X_{\mathcal{L}} \in \mathfrak{X}\left(\mathrm{T}^{2 k-1} Q\right)$ (the Lagrangian vector field) in which the integral curves are holonomic (i.e., they are canonical liftings $j^{2 k-1} \phi: \mathbb{R} \longrightarrow \mathrm{T}^{2 k-1} Q$ of curves $\phi: \mathbb{R} \rightarrow Q$ ) and are the solutions to the Otrogradskii or higher-order Euler-Lagrange equations (see Reference [50-52] for details). 
The Hamilton-Jacobi problem for higher-order Lagrangian dynamical systems is just the slicing problem for the particular situation represented in the diagram

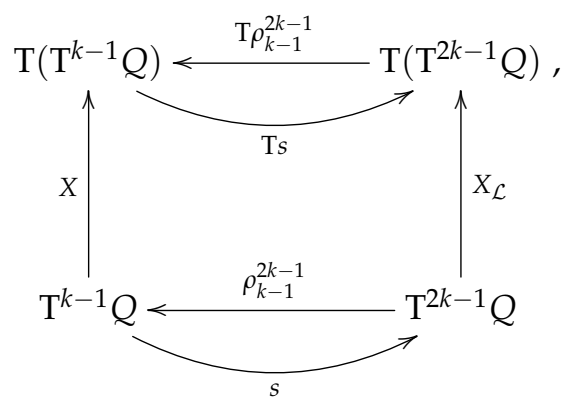

i.e., for sections of the natural projection $\rho_{k-1}^{2 k-1}: \mathrm{T}^{2 k-1} Q \rightarrow \mathrm{T}^{k-1} Q, s \in \Gamma\left(\rho_{k-1}^{2 k-1}\right)$; thus, we have the following settings (see Reference $[20,21]$ for the details and proofs):

Definition 12. The generalized kth-order Lagrangian Hamilton-Jacobi problem for the higher-order Lagrangian system $\left(\mathrm{T}^{2 k-1} Q, \omega_{\mathcal{L}}, E_{\mathcal{L}}\right)$ is to find a section $s \in \Gamma\left(\rho_{k-1}^{2 k-1}\right)$ and $a$ vector field $X \in \mathfrak{X}\left(\mathrm{T}^{k-1} Q\right)$ such that, if $\gamma: \mathbb{R} \rightarrow \mathrm{T}^{k-1} Q$ is an integral curve of $X$, then $s \circ \gamma: \mathbb{R} \longrightarrow \mathrm{T}^{2 k-1} Q$ is an integral curve of $X_{\mathcal{L}}$; i.e., if $X \circ \gamma=\dot{\gamma}$, then $X_{\mathcal{L}} \circ(s \circ \gamma)=\overline{s \circ \gamma}$. Then, the couple $(s, X)$ is a solution to the generalized kth-order Lagrangian HamiltonJacobi problem.

Theorem 8. The following statements are equivalent:

1. The couple $(s, X)$ is a solution to the generalized kth-order Lagrangian Hamilton-Jacobi problem.

2. The vector fields $X$ and $X_{\mathcal{L}}$ are s-related, i.e., $X_{\mathcal{L}} \circ s=\mathrm{Ts} \circ X$. As a consequence, $X=$ $\mathrm{T} \rho_{k-1}^{2 k-1} \circ X_{\mathcal{L}} \circ s$, and $X$ is said to be the vector field associated with the section $s$.

3. The submanifold $\operatorname{Im}(s)$ of $\mathrm{T}^{2 k-1} Q$ is invariant by the Lagrangian vector field $X_{\mathcal{L}}$ (or, which means the same thing, $X_{\mathcal{L}}$ is tangent to $s\left(\mathrm{~T}^{k-1} Q\right)$ ).

4. The integral curves of $X_{\mathcal{L}}$ which have initial conditions in $\operatorname{Im}(s)$ project onto the integral curves of $X$.

5. The equation $i(X)\left(s^{*} \omega_{\mathcal{L}}\right)=\mathrm{d}\left(s^{*} E_{\mathcal{L}}\right)$ holds for $\alpha$.

Proof. (Guidelines for the proof): The proof follows a pattern similar to that of Theorem 1, but now using Definition 12.

Definition 13. The kth-order Lagrangian Hamilton-Jacobi problem for the higher-order Lagrangian system $\left(\mathrm{T}^{2 k-1} Q, \omega_{\mathcal{L}}, E_{\mathcal{L}}\right)$ is to find a section $s \in \Gamma\left(\rho_{k-1}^{2 k-1}\right)$ such that it is a solution to the generalized kth-order Lagrangian Hamilton-Jacobi problem and satisfies that $s^{*} \omega_{\mathcal{L}}=0$. Then, this section $s$ is a solution to the kth-order Lagrangian Hamilton-Jacobi problem.

Observe that that $0=s^{*} \omega_{\mathcal{L}}=-s^{*}\left(\mathrm{~d} \theta_{\mathcal{L}}\right)=-\mathrm{d}\left(s^{*} \theta_{\mathcal{L}}\right)=0$; i.e., $s^{*} \theta_{\mathcal{L}}$ is a closed 1-form and then there exists $S \in \mathrm{C}^{\infty}(\mathcal{U}), U \subset \mathrm{T}^{k-1} Q$, such that $\left.s^{*} \theta_{\mathcal{L}}\right|_{U}=\mathrm{d} S$.

Theorem 9. The following statements are equivalent:

1. The section $s$ is a solution to the generalized kth-order Lagrangian Hamilton-Jacobi problem.

2. $\operatorname{Im}(s)$ is a Lagrangian submanifold of $\mathrm{T}^{2 k-1} Q$, which is invariant by the Lagrangian vector field $X_{\mathcal{L}}$ (and $S$ is a local generating function of this Lagrangian submanifold).

3. The equation $\mathrm{d}\left(s^{*} E_{\mathcal{L}}\right)=0$ holds for $s$, or, which is equivalent, the function $E_{\mathcal{L}} \circ \mathrm{d} S: Q \rightarrow \mathbb{R}$ is locally constant.

Proof. (Guidelines for the proof): They are consequences of Theorem 8 and Definition 13. 
In natural coordinates, from this last condition, we obtain that

$$
\frac{\partial S}{\partial q_{i}^{A}}=\left.\sum_{l=0}^{k-i-1}(-1)^{l} d_{T}^{l}\left(\frac{\partial \mathcal{L}}{\partial q_{i+1+l}^{A}}\right)\right|_{\operatorname{Im}(s)} .
$$

This system of $k n$ partial differential equations for $S$ generalizes Equation (4) to higherorder systems.

Definition 14. Let $\Lambda \subseteq \mathbb{R}^{n}$. A family of solutions $\left\{s_{\lambda} ; \lambda \in \Lambda\right\}$, depending on $n$ parameters $\lambda \equiv\left(\lambda_{1}, \ldots, \lambda_{n}\right) \in \Lambda$, is a complete solution to the $k$ th-order Lagrangian Hamilton-Jacobi problem if the map

$$
\begin{aligned}
\Phi: \mathrm{T}^{k-1} Q \times \Lambda & \longrightarrow \mathrm{T}^{2 k-1} Q \\
(q, \lambda) & \mapsto s_{\lambda}(q)
\end{aligned}
$$

is a local diffeomorphism.

For the Hamiltonian formalism, let $h \in \mathrm{C}^{\infty}\left(\mathrm{T}^{*}\left(\mathrm{~T}^{k-1} Q\right)\right)$ be the Hamiltonian function of a (regular) higher-order dynamical system. Using the canonical Liouville forms of the cotangent bundle, $\theta_{k-1}=p_{A}^{i} \mathrm{~d} q_{i}^{A} \in \Omega^{1}\left(\mathrm{~T}^{*}\left(\mathrm{~T}^{k-1} Q\right)\right)$ and $\omega_{k-1}=\mathrm{d} q_{i}^{A} \wedge \mathrm{d} p_{A}^{i} \in$ $\Omega^{2}\left(\mathrm{~T}^{*}\left(\mathrm{~T}^{k-1} Q\right)\right)$, where $\left(q_{i}^{A}, p_{i}^{A}\right)(1 \leq A \leq n, 0 \leq i \leq k-1)$ are canonical coordinates in $\mathrm{T}^{*}\left(\mathrm{~T}^{k-1} Q\right)$, the dynamical equation for the Hamiltonian system $\left.\left(\mathrm{T}^{*}\left(\mathrm{~T}^{k-1} Q\right), \omega_{k}, h\right)\right)$ is $i\left(X_{h}\right) \omega_{k-1}=\mathrm{d} h$, and it has a unique solution $X_{h} \in \mathfrak{X}\left(\mathrm{T}^{*}\left(\mathrm{~T}^{k-1} Q\right)\right)$. As we are working in the cotangent bundle $\mathrm{T}^{*}\left(\mathrm{~T}^{k-1} Q\right)$, the Hamiltonian Hamilton-Jacobi problems for higherorder systems is stated in the same way as in the first-order case; hence, it is the slicing problem for the particular situation represented in the diagram

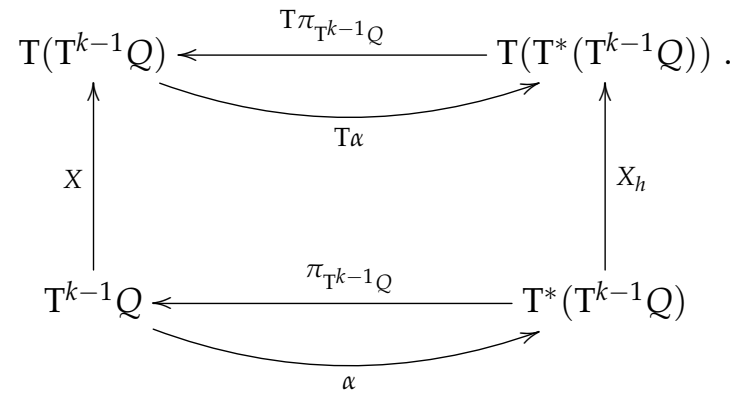

Therefore, all the definitions and results are like in the first-order case, and the relation between both the Lagrangian and the Hamiltonian Hamilton-Jacobi problems is stated as in Theorem 5.

\subsection{Multisymplectic Field Theories}

The Hamilton-Jacobi theory for multisymplectic field theories has been studied in Reference $[42,43,45]$. Next, we state the Lagrangian and the Hamiltonian problems for these systems. For details on multisymplectic field theories, see, for instance, Reference [53-55] and the references therein.

\subsubsection{Multisymplectic Lagrangian Hamilton-Jacobi Problem}

Let $\pi: E \longrightarrow M$ a bundle, where $M$ is an oriented manifold with $\operatorname{dim} M=m$ and $\operatorname{dim} E=n+m$. The Lagrangian description of multisymplectic classical field theories is stated in the first-order jet bundle $\pi^{1}: J^{1} \pi \longrightarrow E$, which is also a bundle $\bar{\pi}^{1}: J^{1} \pi \longrightarrow M$. Natural coordinates in $J^{1} \pi$ adapted to the bundle structure are $\left(x^{i}, y^{\alpha}, y_{i}^{\alpha}\right)(i=1, \ldots, m$; $\alpha=1, \ldots, n)$. Giving a Lagrangian density associated to a Lagrangian function $\mathcal{L}$ and 
using the canonical structures of $J^{1} \pi$, we can define the Poincaré-Cartan forms associated with $\mathcal{L}, \Theta_{\mathcal{L}} \in \Omega^{m}\left(J^{1} \pi\right)$, and $\Omega_{\mathcal{L}}:=-\mathrm{d} \Theta_{\mathcal{L}} \in \Omega^{m+1}\left(J^{1} \pi\right)$, in which local expression is

$$
\Omega_{\mathcal{L}}=-\mathrm{d} \Theta_{\mathcal{L}}=-\mathrm{d}\left(\frac{\partial L}{\partial y_{i}^{\alpha}} \mathrm{d} y^{\alpha} \wedge \mathrm{d}^{m-1} x_{i}-\left(\frac{\partial L}{\partial y_{i}^{\alpha}} y_{i}^{\alpha}-L\right) \mathrm{d}^{m} x\right),
$$

where $\mathrm{d}^{m} x=\mathrm{d} x^{1} \wedge \ldots \wedge \mathrm{d} x^{m}$ and $\mathrm{d}^{m-1} x_{i}=i\left(\frac{\partial}{\partial x^{i}}\right) \mathrm{d}^{m} x$. The Lagrangian function is regular if $\Omega_{\mathcal{L}}$ is a multisymplectic $(m+1)$-form (i.e., 1 -nondegenerate). Then, the couple $\left(J^{1} \pi, \Omega_{\mathcal{L}}\right)$ is a multisymplectic Lagrangian system. The Lagrangian problem consists of finding $m$-dimensional, $\pi^{1}$-transverse, and holonomic distributions $\mathcal{D}_{\mathcal{L}}$ in $J^{1} \pi$ such that their integral sections $\psi_{\mathcal{L}} \in \Gamma\left(\bar{\pi}^{1}\right)$ are canonical liftings $j^{1} \phi$ of sections $\phi \in \Gamma(\pi)$ that are solutions to the Lagrangian field equation

$$
\left(j^{1} \phi\right)^{*} i(X) \Omega_{\mathcal{L}}=0, \text { for every } X \in \mathfrak{X}\left(J^{1} \pi\right) .
$$

In coordinates, the components of $j^{1} \phi=\left(x^{i}, y^{\alpha}, \frac{\partial y^{\alpha}}{\partial x^{i}}\right)$ satisfy the Euler-Lagrange equations

$$
\frac{\partial \mathcal{L}}{\partial y^{A}} \circ j^{1} \phi-\frac{\partial}{\partial x^{\mu}}\left(\frac{\partial \mathcal{L}}{\partial y_{\mu}^{A}} \circ j^{1} \phi\right)=0 .
$$

Definition 15. The generalized Lagrangian Hamilton-Jacobi problem for the multisymplectic Lagrangian system $\left(J^{1} \pi, \Omega_{\mathcal{L}}\right)$ is to find a section $\Psi \in \Gamma\left(\pi^{1}\right)$ (which is called a jet field) and an m-dimensional integrable distribution $\mathcal{D}$ in $E$ such that, if $\gamma \in \Gamma(\pi)$ is an integral section of $\mathcal{D}$, then $\psi_{\mathcal{L}}=\Psi \circ \gamma \in \Gamma\left(\bar{\pi}^{1}\right)$ is an integral section of $\mathcal{D}_{\mathcal{L}}$; i.e., if $\mathrm{T}_{u} \operatorname{Im}(\gamma)=\mathcal{D}_{u}$, for every $u \in \operatorname{Im}(\gamma)$, then $\mathrm{T}_{\bar{u}} \operatorname{Im}(\Psi \circ \gamma)=\left(\mathcal{D}_{\mathcal{L}}\right)_{\bar{u}}$, for every $\bar{u} \in \operatorname{Im}(\Psi \circ \gamma)$. Then, the couple $(\Psi, \mathcal{D})$ is a solution to the generalized Hamiltonian Hamilton-Jacobi problem.

Remark 2. The Hamilton-Jacobi problem can also be stated associating the distributions $\mathcal{D}$ and $\mathcal{D}_{\mathcal{L}}$ with multivector fields. An m-multivector field, on a manifold $\mathcal{M}$ is a section of the bundle $\Lambda^{m}(\mathrm{~T} \mathcal{M}) \rightarrow \mathcal{M}$, where $\Lambda^{m}(\mathrm{~T} \mathcal{M})=\mathrm{T} \mathcal{M} \wedge{ }^{(m)} \wedge \mathrm{T} \mathcal{M}$ (i.e, a skew-symmetric contravariant tensor field). If $\mathbf{X}$ is an m-multivector field in $\mathcal{M}$, then, for every $p \in \mathcal{M}$, there is a neighborhood $U_{p} \subset \mathcal{M}$ and local vector fields $X_{1}, \ldots, X_{m} \in \mathfrak{X}\left(U_{p}\right)$ such that $\left.\mathbf{X}\right|_{U_{p}}=X_{1} \wedge \ldots \ldots . \wedge X_{m}$. Then, if $\mathcal{D}$ is an m-dimensional distribution in $\mathcal{M}$, sections of $\Lambda^{m} \mathcal{D} \rightarrow \mathcal{M}$ are $m$-multivector fields in $\mathcal{M}$, and a multivector field is integrable if its associated distribution is also.

Now, if $\mathcal{M}=J^{1} \pi$, let $\mathbf{X}$ and $\mathbf{X}_{\mathcal{L}}$ be the m-multivector fields associated with the distributions $\mathcal{D}$ and $\mathcal{D}_{\mathcal{L}}$, respectively, then, the Lagrangian Hamilton-Jacobi problem can be represented by the diagram

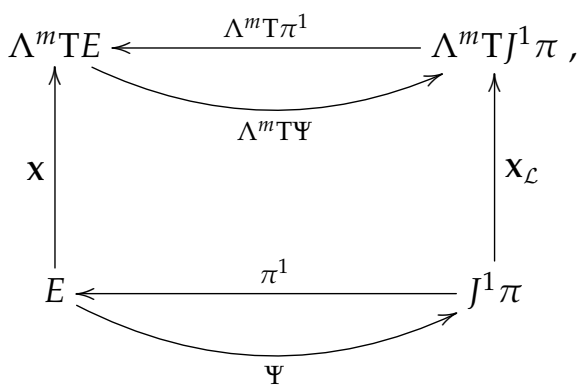

where $\Lambda^{m} \mathrm{~T} \Psi$ and $\Lambda^{m} \mathrm{~T} \pi^{1}$ denote the natural extensions of the maps $\psi$ and $\pi^{1}$ to the multitangent bundles; thus, this problem can be considered as a special case of a slicing problem. 
Theorem 10. The following statements are equivalent:

1. The couple $(\Psi, \mathcal{D})$ is a solution to the generalized Lagrangian Hamilton-Jacobi problem.

2. The distributions $\mathcal{D}$ and $\mathcal{D}_{\mathcal{L}}$ are $\Psi$-related. As a consequence, $\mathcal{D}=\mathrm{T} \pi^{1}\left(\left.\mathcal{D}_{\mathcal{L}}\right|_{\operatorname{Im}(\Psi)}\right)$ and is called the distribution associated with $\Psi$.

3. The distribution $\mathcal{D}_{\mathcal{L}}$ is tangent to the submanifold $\operatorname{Im}(\Psi)$ of $J^{1} \pi$.

4. Integral sections of $\mathcal{D}_{\mathcal{L}}$ which have boundary conditions in $\operatorname{Im}(\Psi)$ project onto the integral sections of $\mathcal{D}$.

5. If $\gamma$ is an integral section of the distribution $\mathcal{D}$ associated with the jet field $\Psi$, then, for every $Y \in \mathfrak{X}(E)$, the equation $\gamma^{*} i(Y)\left(\Psi^{*} \Omega_{\mathcal{L}}\right)=0$ holds for $\Psi$.

Proof. (Guidelines for the proof):

The equivalence between 1 and 2 is a consequence of the Definition 15, the equivalence between distributions and multivector fields, and the definition of integral sections.

Items 3 and 4 follow from 2.

Item 5 is obtained from Definition 15 and using field Equation (10).

Definition 16. The Lagrangian Hamilton-Jacobi problem for the multisymplectic Lagrangian system $\left(J^{1} \pi, \Omega_{\mathcal{L}}\right)$ is to find a jet field $\Psi \in \Gamma\left(\pi^{1}\right)$ such that it is solution to the generalized Lagrangian Hamilton-Jacobi problem and satisfies that $\Psi^{*} \Omega_{\mathcal{L}}=0$. Then, the jet field $\Psi$ is a solution to the Lagrangian Hamilton-Jacobi problem.

The condition $\Psi^{*} \Omega_{\mathcal{L}}=-\mathrm{d}\left(\Psi^{*} \Theta_{\mathcal{L}}\right)=0$ is equivalent to asking that the form $\Psi^{*} \Theta_{\mathcal{L}}$ is closed and then there exists a $(m-1)$-form $\omega \in \Omega^{m-1}(U)$, with $U \subset E$, such that $\Psi^{*} \Theta_{\mathcal{L}}=\mathrm{d} \omega$. Furthermore, $\omega$ is $\pi$-semibasic, since $\Theta_{\mathcal{L}}$, and, hence, $\Psi^{*} \Theta_{\mathcal{L}}$, are also.

Theorem 11. The following statements are equivalent:

1. The jet field $\Psi$ is a solution to the Lagrangian Hamilton-Jacobi problem.

2. $\operatorname{Im}(\Psi)$ is an $m$-Lagrangian submanifold of $J^{1} \pi$ and the distribution $\mathcal{D}_{\mathcal{L}}$ is tangent to it.

3. The form $\Psi^{*} \Theta_{\mathcal{L}}$ is closed. the form

In coordinates, $\omega=W^{i} \mathrm{~d}^{m-1} x_{i}$, and the Lagrangian Hamilton-Jacobi equation has

$$
\sum_{i=1}^{m} \frac{\partial W^{i}}{\partial x^{i}}+\psi_{i}^{\alpha} \frac{\partial W^{i}}{\partial u^{\alpha}}-L\left(x^{i}, u^{\alpha}, \psi_{i}^{\alpha}\right)=0 .
$$

Definition 17. Let $\Lambda \subseteq \mathbb{R}^{m n}$. A family of solutions $\left\{\Psi_{\lambda} ; \lambda \in \Lambda\right\}$, depending on $n$ parameters $\lambda \equiv\left(\lambda_{1}, \ldots, \lambda_{n}\right) \in \Lambda$, is a complete solution to the Lagrangian Hamilton-Jacobi problem if the map

$$
\begin{aligned}
& \Phi: E \times \Lambda \longrightarrow J^{1} \pi \\
& (p, \lambda) \mapsto \Psi_{\lambda}(p)
\end{aligned}
$$

is a local diffeomorphism.

A complete solution defines an $(m-n)$-dimensional foliation in $J^{1} \pi$ which is transverse to the fibers and such that the distribution $\mathcal{D}_{\mathcal{L}}$ is tangent to it. Then, all the sections which are solutions to the Euler-Lagrange equations (i.e., all the integral sections of the distribution $\mathcal{D}_{\mathcal{L}}$ ) are recovered from a complete solution.

\subsubsection{Multisymplectic Hamiltonian Hamilton-Jacobi Problem}

The Hamiltonian formalism for a regular first-order multisymplectic field theory is developed in the so-called reduced dual jet bundle of $J^{1} \pi, J^{1} \pi^{*}=\Lambda_{2}^{m}\left(\mathrm{~T}^{*} E\right) / \Lambda_{1}^{m}\left(\mathrm{~T}^{*} E\right)$, where $\Lambda_{2}^{m}\left(\mathrm{~T}^{*} E\right)$ is the bundle of $m$-forms over $E$ vanishing when they act on $\pi$-vertical bivectors. It is endowed with the canonical projections $\pi_{E}: J^{1} \pi^{*} \rightarrow E$ and $\bar{\pi}_{E}: J^{1} \pi^{*} \rightarrow M$, and natural coordinates in $J^{1} \pi^{*}$ are denoted $\left(x^{i}, y^{\alpha}, p_{\alpha}^{i}\right)$. The physical information is given by a Hamiltonian section $h$ of the natural projection $\mu: \Lambda_{2}^{m}\left(\mathrm{~T}^{*} E\right) \rightarrow J^{1} \pi^{*}$, which 
is associated with a local Hamiltonian function $H \in C^{\infty}\left(J^{1} \pi^{*}\right)$ such that $h\left(x^{i}, y^{\alpha}, p_{\alpha}^{i}\right)=$ $\left(x^{i}, y^{\alpha},-H, p_{\alpha}^{i}\right)$. Then, from the canonical form $\Omega \in \Omega^{m+1}\left(\Lambda_{2}^{m}\left(T^{*} E\right)\right)$, we construct the Hamilton-Cartan multisymplectic form $\Omega_{h}=h^{*} \Omega \in \Omega^{m+1}\left(J^{1} \pi^{*}\right)$ in which coordinate expression is

$$
\Omega_{h}=-\mathrm{d} p_{\alpha}^{i} \wedge \mathrm{d} y^{\alpha} \wedge \mathrm{d}^{m-1} x_{i}+\mathrm{d} H \wedge \mathrm{d}^{m} x,
$$

and the couple $\left(J^{1} \pi^{*}, \Omega_{h}\right)$ is a multisymplectic Hamiltonian system. Then, the Hamiltonian problem consists of finding integrable $m$-dimensional $\bar{\pi}_{E}$-transverse distributions $\mathcal{D}_{h}$ in $J^{1} \pi^{*}$ such that their integral sections $\psi_{h} \in \Gamma\left(\bar{\pi}_{E}\right)$ are solutions to the Hamiltonian field equation

$$
\psi_{h}^{*} i(X) \Omega_{h}=0, \text { for every } X \in \mathfrak{X}\left(J^{1} \pi^{*}\right) .
$$

The existence of such distributions $\mathcal{D}_{h}$ is assured. In coordinates, this equation gives the Hamilton-De Donder-Weyl equations

$$
\frac{\partial\left(y^{A} \circ \psi_{h}\right)}{\partial x^{v}}=\frac{\partial \mathrm{h}}{\partial p_{A}^{v}} \circ \psi_{h} \quad, \quad \frac{\partial\left(p_{A}^{v} \circ \psi_{h}\right)}{\partial x^{v}}=-\frac{\partial \mathrm{h}}{\partial y^{A}} \circ \psi_{h} .
$$

Definition 18. The generalized Hamiltonian Hamilton-Jacobi problem for the multisymplectic Hamiltonian system $\left(J^{1} \pi^{*}, \Omega_{h}\right)$ is to find a section $s \in \Gamma\left(\pi_{E}\right)$ and an integrable mdimensional distribution $\mathcal{D}$ in $E$ such that, if $\gamma \in \Gamma(\pi)$ is an integral section of $\mathcal{D}$, then $\psi_{h}=s \circ \gamma \in \Gamma\left(\bar{\pi}_{E}\right)$ is an integral section of $\mathcal{D}_{h}$, i.e., if $\operatorname{T}_{u} \operatorname{Im}(\gamma)=\mathcal{D}_{u}$, for every $u \in \operatorname{Im}(\gamma)$, then $\operatorname{T}_{\bar{u}} \operatorname{Im}(s \circ \gamma)=\left(\mathcal{D}_{h}\right)_{\bar{u}}$, for every $\bar{u} \in \operatorname{Im}(s \circ \gamma)$. Then, the couple $(s, \mathcal{D})$ is a solution to the generalized Hamiltonian Hamilton-Jacobi problem.

Remark 3. As in the Lagrangian case, the Hamiltonian Hamilton-Jacobi problem can be considered as a special case of the following slicing problem:

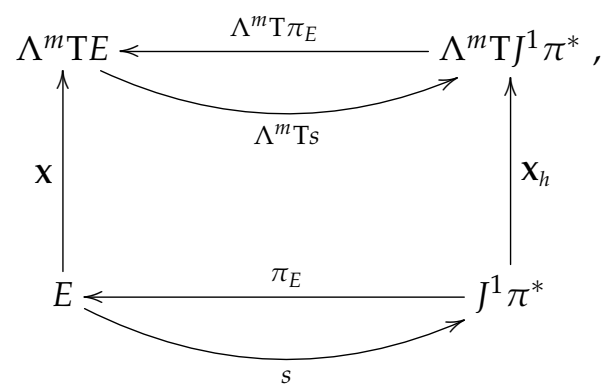

where $\mathbf{X}$ and $\mathbf{X}_{h}$ are m-multivector fields associated with the distributions $\mathcal{D}$ and $\mathcal{D}_{h}$, respectively.

The following Theorems and Definitions are analogous to those of the Lagrangian case.

Theorem 12. The following conditions are equivalent.

1. The couple $(s, \mathcal{D})$ is a solution to the generalized Hamiltonian Hamilton-Jacobi problem.

2. The distributions $\mathcal{D}$ and $\mathcal{D}_{h}$ are s-related. As a consequence, the distribution $\mathcal{D}$ is given by $\mathcal{D}=\mathrm{T} \pi_{E}\left(\left.\mathcal{D}_{h}\right|_{\operatorname{Im}(s)}\right)$, and it is called the distribution associated with $s$.

3. The distribution $\mathcal{D}_{h}$ is tangent to the submanifold $\operatorname{Im}(s)$ of $J^{1} \pi^{*}$.

4. Integral sections of $\mathcal{D}_{h}$ which have boundary conditions in $\operatorname{Im}(s)$ project onto the integral sections of $\mathcal{D}$.

5. If $\gamma$ is an integral section of the distribution $\mathcal{D}$ associated with s, then, for every $Y \in \mathfrak{X}(E)$, the equation $\gamma^{*} i(Y) \mathrm{d}(h \circ s)=0$ holds for $s$.

Definition 19. The Hamiltonian Hamilton-Jacobi problem for the multisymplectic Hamiltonian system $\left(J^{1} \pi^{*}, \Omega_{h}\right)$ is to find a section $s \in \Gamma\left(\pi_{E}\right)$ such that it is a solution to the generalized Hamilton-Jacobi problem and satisfies that $s^{*} \Omega_{h}=0$. The section $s$ is a solution to the Hamiltonianian Hamilton-Jacobi problem. 
Theorem 13. The following conditions are equivalent.

1. The couple $(s, \mathcal{D})$ is a solution to the generalized Hamiltonian Hamilton-Jacobi problem.

2. $\operatorname{Im}(s)$ is an $m$-Lagrangian submanifold of $J^{1} \pi^{*}$ and the distribution $\mathcal{D}_{h}$ is tangent to it.

3. The form $h \circ s \in \Omega^{m}(E)$ is closed.

As the $\pi_{E}$-semibasic $m$-form $h \circ s$ is closed, there exists a local $\pi$-semibasic $(m-1)$ form $\omega \in \Omega^{m-1}(E)$, such that $h \circ s=\mathrm{d} \omega$. In coordinates, if $\omega=W^{i} \mathrm{~d}^{m-1} x_{i}$, where $W^{i} \in$ $C^{\infty}(E)$ are local functions, we obtain that

$$
-H\left(x^{i}, y^{\alpha}, s_{\alpha}^{i}\right)=\sum_{i=1}^{m} \frac{\partial W^{i}}{\partial x^{i}} ; \frac{\partial W^{i}}{\partial y^{\alpha}}=s_{\alpha}^{i},
$$

from which we obtain the classical Hamiltonian Hamilton-Jacobi equation

$$
\sum_{i=1}^{m} \frac{\partial W^{i}}{\partial x^{i}}+H\left(x^{i}, y^{\alpha}, \frac{\partial W^{i}}{\partial y^{\alpha}}\right)=0
$$

The definition and the characteristics of complete solution are like in the Lagrangian case.

\subsubsection{Relation between the Multisymplectic Hamilton-Jacobi Problems}

Let $\mathcal{F} \mathcal{L}: J^{1} \pi \rightarrow J^{1} \pi^{*}$ be the Legendre map defined by the Lagrangian $\mathcal{L}$, which is locally given by

$$
\mathcal{F} \mathcal{L}^{*} x^{i}=x^{i} \quad, \quad \mathcal{F} \mathcal{L}^{*} y^{\alpha}=y^{\alpha} \quad, \quad \mathcal{F} \mathcal{L}^{*} p_{\alpha}^{i}=\frac{\partial \mathcal{L}}{\partial y_{i}^{\alpha}} .
$$

If $\mathcal{L}$ is a regular or a hyperregular Lagrangian (i.e., $\mathcal{F} \mathcal{L}$ is a local or global diffeomorphism), then $\mathcal{F} \mathcal{L}^{*} \Theta_{h}=\Theta_{\mathcal{L}}$ and $\mathcal{F} \mathcal{L}^{*} \Omega_{h}=\Omega_{\mathcal{L}}$. In addition, the integral sections of the distributions $\mathcal{D}_{\mathcal{L}}$ and $\mathcal{D}_{h}$, which are the solution to the Lagrangian and the Hamiltonian problems, respectively, are in one-to-one correspondence through $\mathcal{F} \mathcal{L}$. (see Reference [43] for definitions and details). Then, we have:

Theorem 14. Let $\mathcal{L} \in \Omega^{m}\left(J^{1} \pi\right)$ be a regular or a hyperregular Lagrangian. Then, if $\Psi \in \Gamma\left(\pi^{1}\right)$ is a jet field solution to the (generalized) Lagrangian Hamilton-Jacobi problem, then the section $s=\mathcal{F} \mathcal{L} \circ \Psi \in \Gamma\left(\pi_{E}\right)$ is a solution to the (generalized) Hamiltonian Hamilton-Jacobi problem. Conversely, if $s \in \Gamma\left(\pi_{E}\right)$ is a solution to the (generalized) Hamiltonian Hamilton-Jacobi problem, then the jet field $\Psi=\mathcal{F} \mathcal{L}^{-1} \circ s \in \Gamma\left(\pi^{1}\right)$ is a solution to the (generalized) Lagrangian HamiltonJacobi problem.

Proof. (Guidelines for the proof): The proof is like in Theorem 5, but using multivector fields.

Remark 4. As a final remark, notice that the Hamilton-Jacobi theory for non-autonomous (i.e., time-dependent) dynamical systems can be recovered from the multisymplectic Hamilton-Jacobi theory as a particular case taking $M=\mathbb{R}$ and identifying the distributions $\mathcal{D}, \mathcal{D}_{\mathcal{L}}, \mathcal{D}_{h}$ and their associated multivector fields $\mathbf{X}, \mathbf{X}_{\mathcal{L}}, \mathbf{X}_{h}$ with time-dependent vector fields (see Reference [43]).

\section{Discussion}

In this work, the Lagrangian and the Hamiltonian versions of the Hamilton-Jacobi theory are reviewed from a modern geometric perspective.

First, this formulation is done for autonomous dynamical systems, and, in particular, the Hamiltonian case is compared with the "classical" Hamiltonian Hamilton-Jacobi theory, which is based in using canonical transformations.

There is also a general framework for the theory, which is also reviewed in the work. It contains the above standard theory for autonomous dynamical systems as a particular 
case and allows us to extend the Hamilton-Jacobi theory to a wide range of physical systems. In particular, two of these extensions have been analyzed here: the higherorder (autonomous) dynamical systems and the (first-order) classical Lagrangian and Hamiltonian field theories, using their multisymplectic formulation.

This geometric model has been extended and applied to many kinds of physical systems (as it is mentioned in the Introduction and cited in the bibliography). As a future line of research that has not been explored yet, the application of this geometric framework to state the Hamilton-Jacoby equation for dissipative systems in classical field theories should be explored, using an extension of the contact formalism which has been recently introduced to describe geometrically these kinds of dissipative field theories [56,57].

Funding: I acknowledge the financial support from project PGC2018-098265-B-C33 of the Spanish Ministerio de Ciencia, Innovación y Universidades and the project 2017-SGR-932 of the Secretary of University and Research of the Ministry of Business and Knowledge of the Catalan Government.

Institutional Review Board Statement: Not applicable.

Informed Consent Statement: Not applicable.

Data Availability Statement: Not applicable.

Conflicts of Interest: The author declares no conflict of interest.

\section{References}

1. Arnold, V.I. Mathematical Methods of Classical Mechanics; Graduate Texts in Mathematics; Springer: New York, NY, USA, 1989; Volume 60. [CrossRef]

2. José, J.V.; Saletan, E.J. Classical Dynamics: A Contemporary Approach; Cambridge University Press: Cambridge, UK, 1998; ISBN 9780521636360.

3. Landau, L.; Lifschitz, E. Mécanique, 3rd ed.; Butterworth-Heinemann: Oxford, UK, 1976; Volume 1, ISBN 978-0-7506-2896-9.

4. Saletan, E.J.; Cromer, A.H. Theoretical Mechanics; J. Wiley: New York, NY, USA, 1971; ISBN 13:9780471749868.

5. Dominici, D.; Gomis, J.; Longhi, G.; Pons, J.M. Hamilton-Jacobi Theory For Constrained Systems. J. Math. Phys. 1984, 25 2439-2460. [CrossRef]

6. Constantelos, G.C. On the Hamilton-Jacobi Theory with Derivatives of Higher Order. Il Nuovo Cimento B (1971-1996) 1984, 84, 91-101. [CrossRef]

7. Crandall, M.G.; Evans, L.C.; Lions, P.L. Some properties of viscosity solutions of Hamilton-Jacobi equations. Trans. Am. Math. Soc. 1984, 282, 487-502. [CrossRef]

8. Crandall, M.G.; Lions, P.L. Viscosity solutions of Hamilton-Jacobi equations. Trans. Am. Math. Soc. 1983, 277, 1-42. [CrossRef]

9. Abraham, R.; Marsden, J.E. Foundations of Mechanics, 2nd ed.; Benjamin/Cummings: Reading, MA, USA, 1978. [CrossRef]

10. Benenti, S.; Tulczyjew, W.M. The geometrical meaning and globalization of the the Hamilton-Jacobi method, Differential Geometric Methods in Mathematical Physics. In Differential Geometrical Methods in Mathematical Physics; Springer: Berlin/Heidelberg, Germany, 1980; Volume 836, pp 9-21. [CrossRef]

11. Esposito, G.; Marmo, G.; Sudarshan, G. From Classical to Quantum Mechanics; Cambridge University Press: Cambridge, UK, 2004. . [CrossRef]

12. Libermann, P.; Marle, C.M. Symplectic Geometry and Analytical Dynamics; D. Reidel Publishing Company: Dordrecht, The Netherlands, 1987. [CrossRef]

13. Marmo, G.; Morandi, G.; Mukunda, N. The Hamilton-Jacobi theory and the analogy between classical and quantum mechanics. J. Geom. Mech. 2009, 1, 317-355. [CrossRef]

14. Cariñena, J.F..; Gràcia, X.; Marmo, G.; Martínez, E.; Muñoz-Lecanda, M.C.; Román-Roy, N. Geometric Hamilton-Jacobi theory. Int. J. Geom. Meth. Mod. Phys. 2006, 3, 1417-1458. [CrossRef]

15. Krupková, O.; Vondra, A. On some integration methods for connections on fibered manifolds. In Proceedings of the Conference in Differential Geometry and Its Applications, Opava, Czech Republic, 24-28 August 1992; Silesian University: Opava, Czech Republic, 1993.

16. Cariñena, J.F.; Gràcia, X.; Marmo, G.; Martínez, E.; Muñoz-Lecanda, M.C.; Román-Roy, N. Hamilton-Jacobi theory and the evolution operator. In Mathematical Physics and Field Theory. Julio Abad, in Memoriam; Asorey, M., García Esteve, J.V., Rañada, M.F., Sesma, J., Eds.; Prensas Universitarias de Zaragoza: Zaragoza, Spain, 2009; pp. 177-186, ISBN 978-84-92774-04-3.

17. de León, M.; Martín de Diego, D.; Vaquero, M. A Hamilton-Jacobi theory for singular Lagrangian systems in the Skinner and Rusk setting. Int. J. Geom. Meth. Mod. Phys. 2012, 9, 1250074. [CrossRef]

18. de León, M.; Martń de Diego, D.; Vaquero, M. A Hamilton-Jacobi theory on Poisson manifolds. J. Geom. Mech. 2014, 6, 121-140. [CrossRef] 
19. Leok, M.; Ohsawa, T.; Sosa, D. Hamilton-Jacobi theory for degenerate Lagrangian systems with holonomic and nonholonomic constraints. J. Math. Phys. 2012, 53, 072905.[CrossRef]

20. Colombo, L.; de León, M.; Román-Roy, N.; Prieto-Martínez, P.D. Geometric Hamilton-Jacobi theory for higher-order autonomous systems. J. Phys. A Math. Gen. 2014, 47, 235203. [CrossRef]

21. Colombo, L.; de León, M.; Román-Roy, N.; Prieto-Martínez, P.D. Unified formalism for the generalized kth-order Hamilton-Jacobi problem. Int. J. Geom. Meth. Mod. Phys. 2014, 11, 1460037. [CrossRef]

22. Bates, L.M.; Fassò, F.; Sansonetto, N. The Hamilton-Jacobi equation, integrability, and nonholonomic systems. J. Geom. Mechs. 2014, 6, 441-449. [CrossRef]

23. Cariñena, J.F.; Gràcia, X.; Marmo, G.; Martínez, E.; MUñoz-Lecanda, M.C.; Román-Roy, N. Geometric Hamilton-Jacobi theory for nonholonomic dynamical systems. Int. J. Geom. Meth. Mod. Phys. 2010, 7, 431-454. [CrossRef]

24. de León, M.; Marrero, J.C.; Martín de Diego, D. Linear almost Poisson structures and Hamilton-Jacobi equation, Applications to nonholonomic mechanics. J. Geom. Mech. 2010, 2, 159-198. [CrossRef]

25. Iglesias-Ponte, D.; de León, M.; Martín de Diego, D. Towards a Hamilton-Jacobi theory for nonholonomic mechanical systems. J. Phys. A Math. Theor. 2008, 41, 015205. [CrossRef]

26. Ohsawa, T.; Bloch, A.M.; Leok, M. Nonholonomic Hamilton-Jacobi theory via Chaplygin Hamiltonization. J. Geom. Phys. 2011, 61, 1263-1291. [CrossRef]

27. Ohsawa, T.; Bloch, A.M. Nonholomic Hamilton-Jacobi equation and integrability. J. Geom. Mech. 2011, 1, 461-481. [CrossRef]

28. Barbero-Liñán, M.; de León, M.; Martín de Diego, D.; Marrero, J.C.; Muñoz-Lecanda, M.C. Kinematic reduction and the Hamilton-Jacobi equation. J. Geom. Mech. 2012, 4, 207-237. [CrossRef]

29. Wang, H. Symmetric Reduction and Hamilton-Jacobi Equation of Rigid Spacecraft with a Rotor. J. Geom. Symm. Phys. 2013, 32, 87-111. [CrossRef]

30. Grillo, S.; Padrón, E. A Hamilton-Jacobi Theory for general dynamical systems and integrability by quadratures in symplectic and Poisson manifolds. J. Geom. Phys. 2016, 10, 101-129. [CrossRef]

31. Balseiro, P.; Marrero, J.C.; Martín de Diego, D.; Padrón, E. A unified framework for mechanics: Hamilton-Jacobi equation and applications. Nonlinearity 2010, 23, 1887-1918. [CrossRef]

32. Leok, M.; Sosa, D. Dirac structures and Hamilton-Jacobi theory for Lagrangian mechanics on Lie algebroids. J. Geom. Mechs. 2012, 4, 421-442. [CrossRef]

33. de León, M.; Laínz, M. A review on contact Hamiltonian and Lagrangian systems. arXiv 2020, arXiv:2011.05579.

34. Grillo, S.; Padrón, E. Extended Hamilton-Jacobi theory, contact manifolds, and integrability by quadratures. J. Math. Phys. 2020, 61, 012901. [CrossRef]

35. Barbero-Liñán, M.; de León, M.; Martín de Diego, D. Lagrangian submanifolds and Hamilton-Jacobi equation. Monatshefte Math. 2013, 171, 269-290. [CrossRef]

36. Cariñena, J.F.; Gràcia, X.; Marmo, G.; Martínez, E.; Muñoz-Lecanda, M.C.; Román-Roy, N. Structural aspects of Hamilton-Jacobi theory. Int. J. Geom. Meth. Mod. Phys. 2016, 13, 1650017. [CrossRef]

37. Wang, H. Hamilton-Jacobi theorems for regular reducible Hamiltonian systems on a cotangent bundle. J. Geom. Phys. 2017, 119, 82-102. [CrossRef]

38. Barbero-Liñán, M.; Delgado-Téllez, M.; Martín de Diego, D. A geometric framework for discrete Hamilton-Jacobi equation. Proceedings of the XX International Fall Workshop on Geometry and Physics. Madrid. AIP Conf. Proc. 2012, 1460, 164-168. [CrossRef]

39. Ohsawa, T.; Bloch, A.M.; Leok, M. Discrete Hamilton-Jacobi theory. SIAM J. Control Optim. 2011, 49, 1829-1856. [CrossRef]

40. de León, M.; Martín de Diego, D.; Marrero, J.C.; Salgado, M.; Vilariño, S. Hamilton-Jacobi theory in $k$-symplectic field theories. Int. J. Geom. Meth. Mod. Phys. 2010, 7, 1491-1507. [CrossRef]

41. de León, M.; Vilariño, S. Hamilton-Jacobi theory in k-cosymplectic field theories. Int. J. Geom. Methods Mod. Phys. 2014, 11, 1450007. [CrossRef]

42. de León, M.; Marrero, J.C.; Martín de Diego, D. A geometric Hamilton-Jacobi theory for classical field theories. In Variations, Geometry and Physics; Nova Science Publisher: New York, NY, USA, 2009; pp. 129-140. Available online: http://hdl.handle.net/ 10261/4169 (accessed on 1 August 2008).

43. De León, M.; Prieto-Martínez, P.D.; Román-Roy, N.; Vilariño, S. Hamilton-Jacobi theory in multisymplectic classical field theories. J. Math. Phys. 2017, 58, 092901. [CrossRef]

44. Vitagliano, L. The Hamilton-Jacobi formalism for higher-order field theory. Int. J. Geom. Meth. Mod. Phys. 2010, 7, 1413-1436. [CrossRef]

45. Vitagliano, L. Geometric Hamilton-Jacobi field theory. Int. J. Geom. Methods Mod. Phys. 2012, 9, 1260008. [CrossRef]

46. Campos, C.M.; de León, M.; Martín de Diego, D.; Vaquero, M. Hamilton-Jacobi theory in Cauchy data space. Rep. Math. Phys. 2015, 76, 359-387. [CrossRef]

47. Vitagliano, L. Hamilton-Jacobi diffieties. J. Geom. Phys. 2011, 61, 1932-1949. [CrossRef]

48. Vitagliano, L. Characteristics, bicharacteristics, and geometric singularities of solutions of PDEs. Int. J. Geom. Meth. Mod. Phys. 2014, 11, 1460039. [CrossRef]

49. Crampin, M. Tangent bundle geometry for Lagrangian dynamics. J. Phys. A Math. Gen. 1983, 16, 3755-3772. [CrossRef] 
50. de León, M.; Rodrigues, P.R. Generalized Classical Mechanics and Field Theory; North-Holland Math. Studies; Elsevier Science Publishers B.V.: Amsterdam, The Netherlands, 1985; Volume 112, ISBN 9780444877536.

51. Gràcia, X.; Pons, J.M.; Román-Roy, N. Higher-order Lagrangian systems: Geometric structures, dynamics and constraints. J. Math. Phys. 1991, 32, 2744-2763. [CrossRef]

52. Prieto-Martínez, P.D.; Román-Roy, N. Lagrangian-Hamiltonian unified formalism for autonomous higher-order dynamical systems. J. Phys. A Math. Theor. 2011, 44, 385203. [CrossRef]

53. Cariñena, J.F.; Crampin, M.; Ibort, L.A. On the multisymplectic formalism for first order field theories. Differ. Geom. Appl. 1991, 1, 345-374. [CrossRef]

54. Echeverría-Enríquez, A.; Muñoz-Lecanda, M.C.; Román-Roy, N. Geometry of Lagrangian first-order classical field theories. Fortschr. Phys. 1996, 44, 235-280. [CrossRef]

55. Román-Roy, N. Multisymplectic Lagrangian and Hamiltonian formalisms of classical field theories. Symmetry Integr. Geom. Methods Appl. (SIGMA) 2009, 5, 25. [CrossRef]

56. Gaset, J.; Gràcia, X.; Muñoz-Lecanda, M.; Rivas, X. Román-Roy, N. A contact geometry framework for field theories with dissipation. Ann. Phys. 2020, 414, 168092. [CrossRef]

57. Gaset, J.; Gràcia, X.; Muñoz-Lecanda, M.; Rivas, X.; Román-Roy, N. A $k$-contact Lagrangian formulation for nonconservative field theories. arXiv 2020, arXiv:2002.10458. 